Please quote as: Meis, J.; Menschner, P. \& Leimeister, J. M. (2010): Modellierung von Dienstleistungen mittels Business Service Blueprinting Modeling. In: Dienstleistungsmodellierung: Interdisziplinäre Konzepte und Anwendungsszenarien Proceedings der Dienstleistungsmodellierung 2010, Workshop im Rahmen der Modellierung 2010, Heidelberg, Germany. 


\section{Modellierung von Dienstleistungen mittels Business Service Blueprinting Modeling}

Jochen Meis, Philipp Menschner und Jan Marco Leimeister

Der Beitrag stellt mit Business Service Blueprint Modeling (BSBM) einen konzeptionellen Ansatz zur Modellierung von Dienstleistungen vor, der ein systematisches und standardisiertes Vorgehen zur Modellierung von Dienstleistungen mit Kundeninteraktion anstrebt. BSBM verwendet hierzu die Business Process Modeling Notation (BPMN) in einem Service Blueprint (SB). Somit wird ein Modellierungskonzept geschaffen, das sowohl Kundeninteraktionspunkte berücksichtigt, als auch von aktuellen Werkzeugen unterstützt wird. BSBM nutzt Pools und Lanes der BPMN zur Abbildung der Ebenen des Service Blueprints. Zur Definition der Ebenen werden drei Varianten vorgestellt und anhand von Kriterien diskutiert. Die aus der Literatur und Projekten abgeleiteten Kriterien beeinflussen die Einsatzmöglichkeit der Varianten. BSBM als Kombination von SB und BPMN ermöglicht die Visualisierung der Aktivitäten und Kundeninteraktionen bei gleichzeitiger werkzeuggestützten Modellierung der Dienstleistung. Dieses wird am Beispiel der wohnbegleitenden Dienstleistung ,Vitalcheck“ verdeutlicht. Hiermit wird eine standardisierte Modellierung von Dienstleistungsprozessen erreicht, dem ein systematisches Vorgehen zur Erfassung von Kundeninteraktionen zu Grunde gelegt wird.

\section{Motivation}

Dienstleistungen dominieren westliche Volkswirtschaften, ca. 70\% der Bruttowertschöpfung und der Arbeitsplätze werden dem tertiären Sektor zugerechnet (Rai und Sambamurthy 2006). Allerdings gestalten sich für Dienstleistungsunternehmen die systematische Entwicklung von Dienstleistungen und die Gestaltung ihres Portfolios schwierig. Nicht nur „reine“ Dienstleistungsanbieter, sondern auch Unternehmen mit produktbegleitenden Dienstleistungen oder hybriden Produkten (Leimeister und Glauner 2008) haben sich geeignete Methoden und Hilfsmittel zur systematischen Entwicklung von Dienstleistungen noch nicht in allen Entwicklungsphasen angeeignet bzw. setzen diese noch nicht ausgiebig ein. Ausgehend von dieser Situation, ist die Dienstleistungsentwicklung bei den Anbietern in der Regel von intuitivem Vorgehen, schöpferischem Phantasieren und Visionieren ge- 
prägt und stellt einen zumeist kreativen Prozess dar (Bullinger und Scheer 2006). Daraus resultiert, dass viele Probleme einer Dienstleistung, vor allem in Bezug auf Akzeptanz und Kostenstruktur, bereits in der Planung und Entwicklung der Dienstleistung entstehen und sich auf die Erbringung auswirken. In Analogie zum Pareto Prinzip werden bei der Dienstleistungsentwicklung $80 \%$ der verursachten Kosten im Erbringungsprozess bereits in den frühen Phasen der Planung festgelegt. Daher müssen Dienstleistungen zielgerichtet analysiert, geplant und realisiert werden.

Zur Entwicklung von Dienstleistungen wird im Service Engineering während der Designphase die Dienstleistung unter Verwendung geeigneter Werkzeuge und Methoden modelliert. Damit Dienstleistungsprozesse verwaltet und organisiert werden können, müssen sie beschrieben und dokumentiert werden. $\mathrm{Zu}$ Beschreibung und Dokumentation von Prozessen existieren verschiedene Möglichkeiten (Scheer und Thomas 2005; Fließ 2006; White 2004). Zur Darstellung wird eine geeignete Methode benötigt, mit der die Zusammenhänge von Ressourcen und Aktivitäten dargestellt werden. Das SB ist eine Option, die die Darstellung von Kundeninteraktionspunkten und von Aktivitäten vom Dienstleister zum Kunden ermöglicht. Eine weitere einfache und standardisierte Modellierung von Prozessen bietet die Business Process Modeling Notation (BPMN). Mithilfe von Werkzeugen lassen sich Dienstleistungsprozesse mittels BPMN modellieren, dokumentieren und evaluieren. Abhängig von dem Werkzeug und der Systemlandschaft können die Modelle in einem Business Process Management System (BPMS) ausgeführt werden.

Durch die Technisierung der Arbeitswelt halten Informations- und Kommunikationstechniken (IKT) immer mehr Einzug in die Erbringung von Dienstleistungen. Somit müssen Dienstleistungsprozesse nicht mehr nur die Interaktion zwischen Kunde und Personal berücksichtigen, sondern zusätzlich IKT Komponenten (Riedl et al. 2009). Des Weiteren wird mit dem vermehrten Einsatz von IKT die Möglichkeit geboten, einzelne Prozessschritte mit IKT zu unterstützen. Eine Möglichkeit hierfür ist die frühzeitige Erfassung von Informationen (z.B. direkt innerhalb der Wohnung) und Überführung der Information ohne Medienbruch in die Dienstleistung.

Obwohl innovative Methoden zur Dienstleistungsentwicklung vereinzelt erfolgreich angewendet werden konnten, trifft das in der Praxis noch nicht auf alle Dienstleistungssektoren zu. Besonders im Bereich der personenbezogenen Dienstleistungen werden IT Potenziale heute noch gar nicht oder nur in geringem Ausmaß genutzt (vgl. hierzu auch die Arbeiten zum Technology Spillover im Dienstleistungssektor (Hsieh und Yuan 2010)). Zu den personenbezogenen Dienstleistungen zählen Tätigkeiten aus der Bildung, Freizeit, gesundheitlichen Versorgung, haushaltsnahe oder wohnbegleitende Dienstleistungen. Gerade hier liegt aber viel ökonomisches Potential verborgen. Dies wird unter anderem bedingt durch den demographischen Wandel, der sich in erhöhten Ausgaben für den Gesundheitssektor als auch in einer steigenden Nachfrage nach haushaltsnahen und wohnbeglei- 
tenden Dienstleistungen verantwortlich zeigt (RWI 2008; Philpott 2000; Pötzsch und Sommer 2006).

Jedoch gestaltet sich eine systematische Modellierung und Entwicklung von Dienstleistungen im Bereich der personenbezogenen Dienstleistungen besonders schwierig. Hierzu zählen auch die wohnbegleitenden Dienstleistungen, die innerhalb der Wohnung des Kunden an ihm selbst (z.B. frisieren zu Hause) oder seiner Wohnung (z.B. Fensterreinigung) erbracht werden (Meis und Draeger 2007).

Diese sind größtenteils geprägt durch den direkten und persönlichen Kontakt zwischen Kunde und Dienstleister, jedoch erhält auch in diesen Sektoren IKT verstärkt Einzug. Es besteht daher Bedarf an einer Methode, die die zwei Aspekte (Persönlicher Kontakt und Einsatz von IKT) kombiniert.

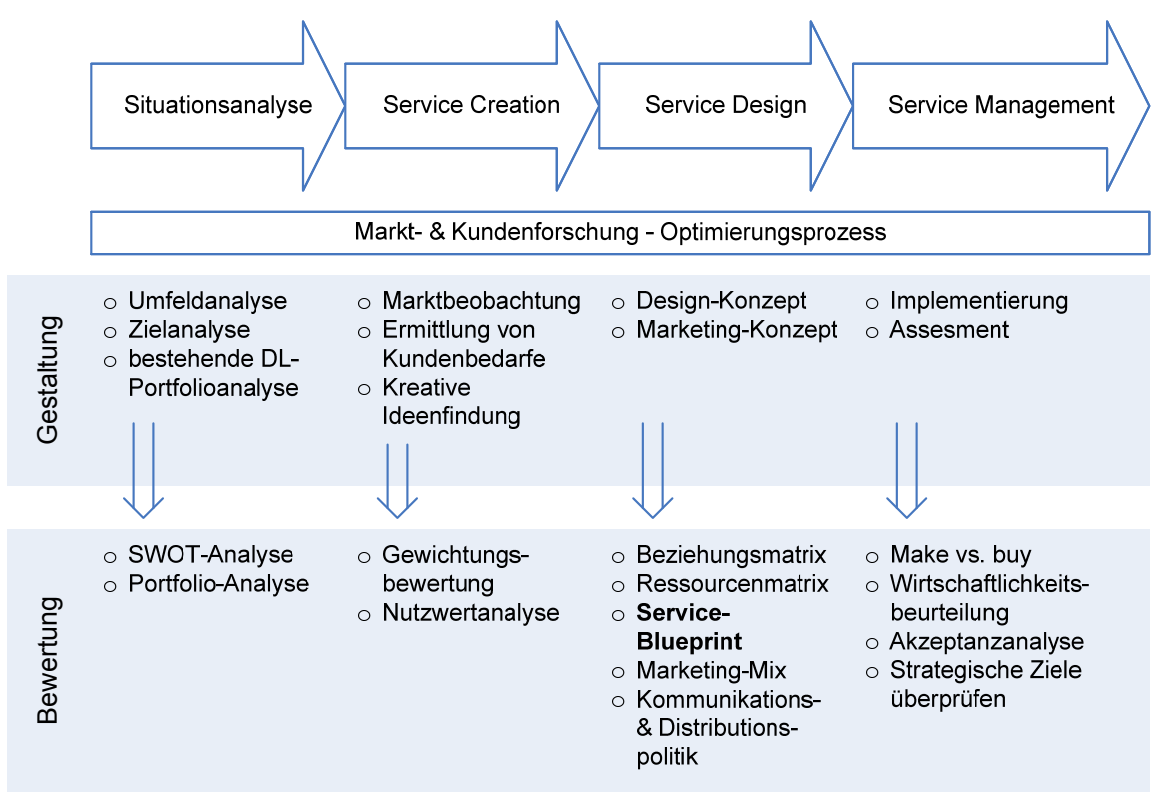

Abb. 1. Vorgehensmodell nach Hohm et al. (eigene Darstellung in Anlehnung an (Hohm et al. 2004))

Der Lebenszyklus einer Dienstleistung umfasst mehrere Phasen. Im Rahmen des Service Engineerings können aus verschiedenen Vorgehensmodellen zur Entwicklung von Dienstleistungen vier Phasen differenziert werden: Analyse, Ideengenerierung, Design und Umsetzung sowie Erbringung. Teilweise weichen Vorgehensmodelle von diesen Phasen ab, in dem sie Phasen zusammenfassen oder weiter detaillieren (Hohm et al. 2004; Schneider et al. 2003; Meiren et al. 1998). Zur Einordnung des Service Blueprints in den Entwicklungsprozess einer Dienstleistung wird hier exemplarisch das 4-Phasen Vorgehensmodell nach Hohm et al. vorgestellt. Dieses Vorgehensmodell ist speziell für die Wohnungswirtschaft entwickelt worden und gibt ausgewählte Methoden zur Unterstützung der Phasen an. 
Das SB wird in der Phase des Service Designs zur Darstellung von Merkmalsdimensionen und Prozessaktivitäten eingesetzt (Hohm et al. 2004) (siehe Abb. 1).

Im Folgenden wird zu Beginn auf die Anwendungsdomäne der IT-gestützten wohnbegleitenden Dienstleistungen eingegangen. Anschließend werden das SB und die BPMN in seiner Historie vorgestellt und bewertet. Im Anschluss werden Kriterien festgelegt, die zur Bewertung von BSBM herangezogen werden. Abschließend wird BSBM vorgestellt und anhand des Beispiels der wohnbegleitenden Dienstleistung „Vitalcheck“ verdeutlicht und bewertet.

\section{Anwendungsdomäne der IT-gestützten wohnbegleitenden Dienstleistungen}

Der Begriff „Dienstleistung“ wird in der Literatur sehr stark diskutiert und ist einem stetigen Wandel unterzogen. Neuere Konzepte und innovative Techniken sowie die sich wandelnde Gesellschaft prägen und gestalten den Begriff und die Interpretation von Dienstleistungen. Eine konkrete Definition hat sich hingegen noch nicht durchgesetzt, wohl aber ein Konsens, dass drei Dienstleistungsdimensionen (Potential-, Prozess-, Ergebnisdimension) fokussiert werden müssen (Schneider und Thomas 2003; Meffert und Bruhn 2008; Maleri 1997). Ein wichtiger Aspekt bei jeder Betrachtung von Dienstleistungen ist die „Mitwirkung des Kunden“ (Berekoven 1974; Hilke 1989; Meyer 1992; Engelhardt et al. 1993; Zink und Eberhard 2009). Der Kunde bzw. das Kundenobjekt ist der wesentliche externe Faktor, welcher innerhalb des Dienstleistungsprozesses berücksichtigt werden muss.

Über wohnbegleitende Dienstleistungen wird in anderen Zusammenhängen auch von haushaltsnahen Dienstleistungen gesprochen. Die Gemeinsamkeit besteht in dem eindeutigen Bezug der Dienstleistung zum Bewohner, dem Kunden, und seiner Wohnung, dem Kundenobjekt. Derzeit liegt noch keine einheitliche Definition für und Trennung von wohnbegleitenden Dienstleistungen vor (Beuerle und Petter 2008; Scharp et al. 2004). In Zeiten des Überangebots von Wohnungen ist für Wohnungssuchende nicht nur das Wohnungsangebot, sondern auch das wohnbegleitende Dienstleistungsangebot entscheidend (Beuerle und Petter 2008). Die wohnbegleitenden Dienstleistungen werden als Instrument der Service- und Kommunikationspolitik seitens der Wohnungswirtschaft eingesetzt. Zu der Servicepolitik werden beispielsweise Kundeninformationen, Unterstützung bei Vermietung und Verwaltung, Abholdienste, Umzugshilfe usw. gezählt.

Der Einsatz von IT zur Unterstützung des Prozesses wohnbegleitender Dienstleistungen ist der Technisierung unserer Gesellschaft geschuldet. Hierzu sind Dienstleistungen zu IT-gestützten Dienstleistungen und IT-Dienstleistungen abzugrenzen. Anhand der drei Merkmalsdimensionen lassen sich IT-Dienstleistungen sehr gut charakterisieren und von IT-gestützten Dienstleistungen abgrenzen (Böhmann 2004). 
IT-gestützte wohnbegleitende Dienstleistungen können im Bereich des Leistungspotenzials oder des Leistungsprozesses durch den Einsatz von IT unterstützt werden. Eine Möglichkeit hierfür ist die Integration und Verwendung von Gebäudeautomationssystemen als Informationslieferant und Kommunikationsschnittstelle. Somit wird ein umfangreiches Angebot von innovativen IT-gestützten wohnbegleitenden Dienstleistungen ermöglicht, die lokale Sensoren und Aktoren in den Dienstleistungsprozess integrieren.

\section{Service Blueprint}

\subsection{Historie}

Die Methode des Service Blueprint (SB) wurde 1984 zur Beschreibung von Dienstleistungsprozessen vorgestellt und in den nachfolgenden Jahren weiterentwickelt (Shostack 1984). Sie ist zum Ausgleich des Mangels von systematischen Methoden zur Dienstleistungsentwicklung eingeführt worden. Die erste Entwicklungsstufe von Shostack unterscheidet zwischen zwei Aktivitätsebenen, die durch die Sichtbarkeitslinie („Line of Visibility“) getrennt sind. Somit sind die Schritte in denen der Kunde an der Dienstleistungserbringung beteiligt ist sichtbar von den notwendigen Aktivitäten zur Dienstleistungserbringung aus Anbietersicht getrennt. Eine weitere Trennung von Kundenaktivitäten zur Interaktion mit dem Anbieter bietet die Kundeninteraktionslinie („Line of Interaction“) an. Die Kundeninteraktionslinie verdeutlicht die Kommunikation zwischen dem Kunden und dem Anbieter. Es werden somit die Kundenaktivitäten von den Dienstleisteraktivitäten getrennt visualisiert.

In der zweiten Entwicklungsstufe hat Kingman-Brundage weitere Trennlinien zwischen Aktivitäten auf der Anbieterseite eingeführt (Kingman-Brundage 1989). Zum einen trennt die interne Interaktionslinie („Line of internal interaction“) unterstützende Aktivitäten des Anbieters von seinen Aktivitäten im Hintergrund, den sogenannten Backofficeaktivitäten. Unterstützende Aktivitäten werden als Supportaktivitäten bezeichnet, die von unterschiedlichsten Fachabteilungen erbracht werden. Diese werden nicht vom Kunden direkt wahrgenommen (Kleinaltenkamp 2000; Fließ 2006). Zum anderen trennt die Implementierungslinie (,Line of implementation") die Supportaktivitäten von den Managementaktivitäten. Während die Supportaktivitäten zum speziellen Dienstleistungserstellungsprozess gehören, sind die Managementaktivitäten vorbereitende Aktivitäten und besitzen für mehrere Dienstleistungen ihre Gültigkeit. Klassische Managementaktivitäten sind Controlling und Personalplanung (Kleinaltenkamp 2000; Fließ 2006).

Die dritte Entwicklungsstufe nach Fließ und Kleinaltenkamp (2004) bietet die Möglichkeit der Abgrenzung des Leistungserstellungsprozesses vom Leistungspotenzial durch eine zusätzliche Vorplanungslinie („Line of order penetration“). Oberhalb der Vorplanungslinie finden die Aktivitäten des Dienstleistungserstellungsprozesses statt. Unterhalb sind hingegen die vorbereitenden Aktivitäten dar- 
gestellt, die für die Bereitstellung des Leistungspotenzials der Dienstleistung verantwortlich sind. Der Einsatz dieser Aktivitäten ist im Vorfeld nur schwer abzuschätzen, da die Integration in die Dienstleistung in Bezug auf Zeit, Dauer und Intensität schwer zu planen ist (Kleinaltenkamp 2000). Folglich bietet das SB in der dritten Entwicklungsstufe die Möglichkeit der visuellen Darstellung dieses Defizits sowie der Differenzierung des Leistungsprozesses vom Leistungspotenzial (Fließ und Kleinaltenkamp 2004; Fließ 2006).

Eine weitere Entwicklungsstufe des Service Blueprints speziell für IT-gestützte wohnbegleitende Dienstleistungen unterteilt die Kundenaktivitäten. Hierbei werden Kundenaktivitäten von selbständig agierenden Kundenaktivitäten unterschieden. Die Kundenaktivitäten werden direkt vom Kunden durchgeführt. Diese können entweder die Kundeninteraktion zum Dienstleister oder die Gebäudeautomation der Wohnung betreffen. Die Gebäudeautomation übernimmt dabei die selbständig agierenden Kundenaktivitäten, welche direkt oder indirekt von den Kundenaktivitäten abhängig sind (Meis und Schöpe 2006). Die selbstständig agierenden Kundenaktivitäten werden durch den Einsatz von IKT ausgeführt. Abb. 2 gibt einen Überblick aller Entwicklungsstufen des Service Blueprints. Sind einzelne Ebenen des Service Blueprints für eine Dienstleistung nicht relevant, da dort beispielsweise keine Aktivitäten stattfinden, so kann diese Ebene bei der Darstellung entfallen.

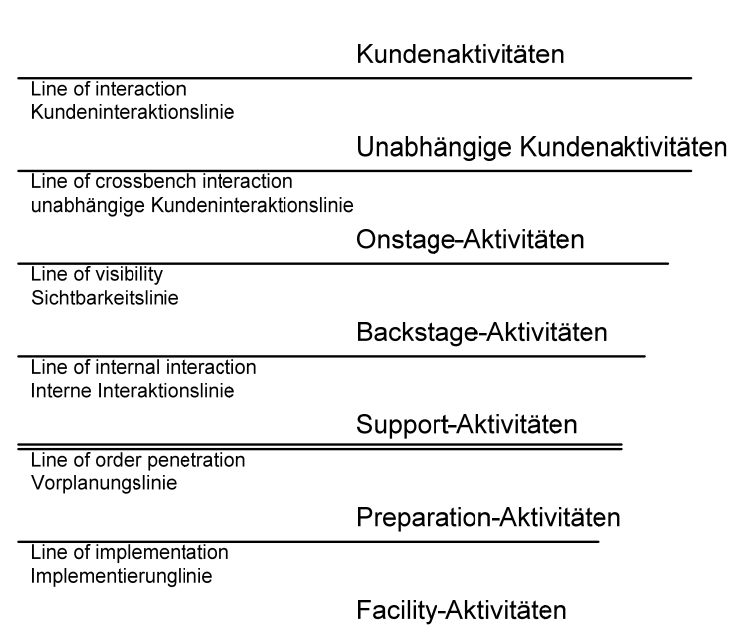

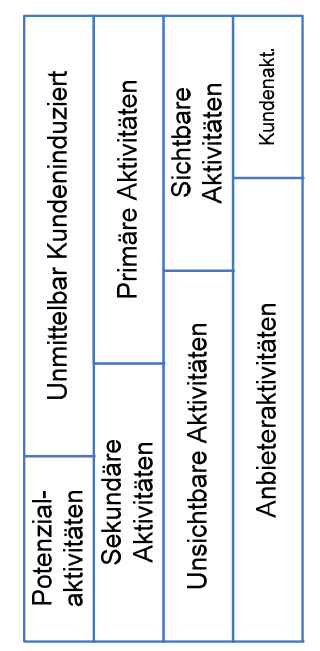

Abb. 2. Struktur des Service Blueprints für IT-gestützte wohnbegleitende Dienstleistungen

Die Erweiterungen für IT-gestützte wohnbegleitende Dienstleistungen umfasst das Potenzial von intelligenten Wohnungen. Die selbständig agierenden Kundenaktivitäten umfassen Informationen von Sensoren und Aktoren innerhalb der Wohnung. Diese sind konstant in der Wohnung aktiv und initiieren nach einem individuell definierten Schema (z.B. Benachrichtigung an den Nachbarn bei Brand- 
erkennung mittels Rauchsensoren) eine Dienstleistung. Daher müssen diese im Dienstleistungsprozess mit berücksichtigt und modelliert werden.

\subsection{Methode}

SB ist eine Methode zur Darstellung, Analyse und Gestaltung von Prozessen, die bei dem Kunden beginnen und enden und einen Mehrwert für den Kunden schaffen. Mit dem SB können Schwachstellen im Dienstleistungsprozess aufgezeigt werden. Über die einzelnen Aktivitäten, die zur Erbringung einer Dienstleistung durchgeführt werden, wird somit ein gemeinsames Verständnis des Leistungsumfangs erreicht. Das SB unterstützt einen Dienstleistungsentwickler nicht nur bei der Identifizierung von Problemen bevor sie eintreten, sondern auch bei der Entwicklung von neuen Marktpotenzialen. Neben der Entwicklung von Dienstleistungen wird das SB auch zum Testen der Qualität einer Dienstleistung eingesetzt (Shostack 1984; Fließ 2006).

Shostack verwendete für das SB eine Notation in Anlehnung an Flussdiagramme. Innerhalb der Literatur sind in das SB weitere unterschiedliche Notationen eingebracht worden. Somit werden die Vorteile der Notation mit den Vorteilen des Service Blueprints für einen bestimmten Einsatzzweck kombiniert. Um einen Überblick der verwendeten Notationen zu geben, wird ein vereinfachter Dienstleistungsprozess zur Fensterreinigung genutzt (siehe Abb. 3).

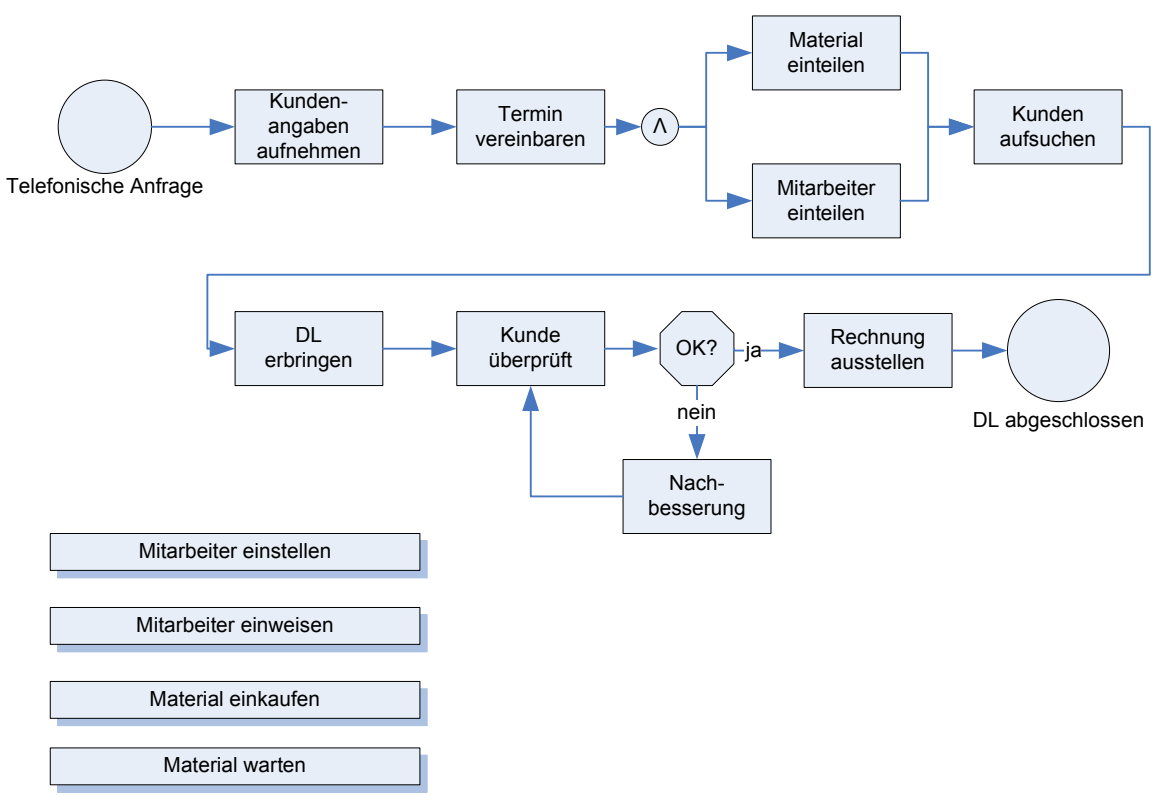

Abb. 3. Dienstleistungsprozess als Beispielprozess (Flussdarstellung) 
Der Kunde ruft bei dem Anbieter an und wählt die angebotene Dienstleistung aus. Nachdem der Umfang der Tätigkeit ermittelt ist, wird anschließend ein Termin für die Dienstleistungserbringung vereinbart.

\section{Notation in Anlehnung an Flussdiagramme}

Eine einfache verständliche Notation für die Service Blueprints ist an die Flussdiagrammdarstellung angelehnt - im Folgenden als Standardnotation bezeichnet (Fließ et al. 2004). Diese Notation unterscheidet zwischen Kunden- und Anbieteraktionen. Zusätzlich werden Entscheidungen und Fehlerpunkte berücksichtigt. Sie bietet eine einfache Vermittlung des Dienstleistungsprozesses mit anderen Gesprächspartnern, um ein gemeinsames Verständnis des Dienstleistungsprozesses zu erhalten. Identifizierte Aktivitäten mit einem hohen Fehlerpotenzial werden in der Darstellung gesondert ausgezeichnet. Der Beispieldienstleistungsprozess lässt sich mit der Standardnotation in SB-Anordnung darstellen (siehe Abb. 4).

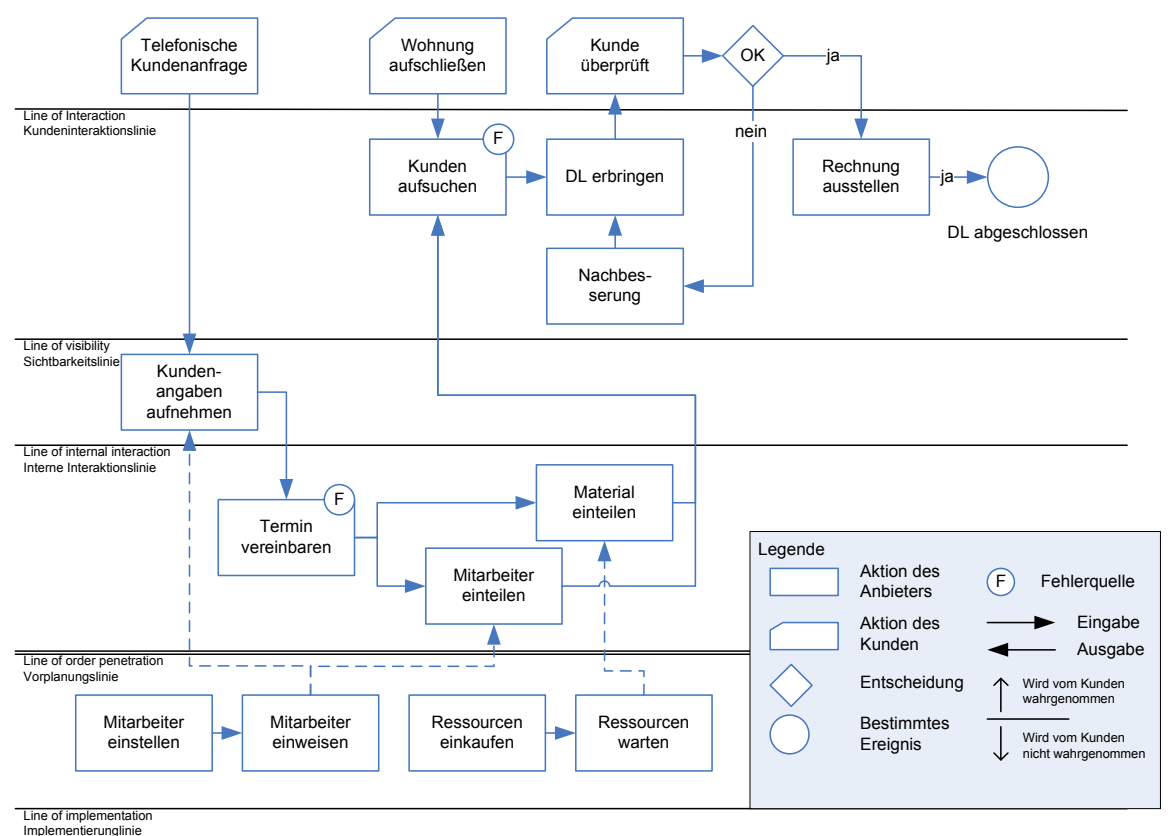

Abb. 4. Beispielprozess in Standardnotation in SB-Anordnung

Die Standardnotation ist eine einfache Flussdiagrammdarstellung. Mit dieser Darstellung können Zusammenhänge und Abfolgen von einzelnen Aktivitäten modelliert werden. Die Flussdiagrammdarstellung existiert in vielen verschiedenen Ausprägungen mit gleichem Paradigma, die sich im Detail bei der Verwendung abgewandelter Symbole unterscheiden. Daher hat sich bisher auch kein 
Standard bzw. Norm für diese Darstellung etabliert. Auch ist eine werkzeuggestützte Ausführbarkeit dieser modellierten Prozesse nicht möglich.

\section{Gantt-Diagramm}

Neben der einfachen Darstellung eines Dienstleistungsprozesses in Standardnotation ist ebenfalls der Einsatz eines Gantt-Diagramms möglich (Fließ et al. 2004). Mit dem Gantt-Diagramm werden zeitliche Abhängigkeiten von einzelnen Aktivitäten verdeutlicht. Hierbei wird der Prozess mittels einer zeitlichen Unterteilung und der Aneinanderreihung von Aktivitäten auf horizontalen Verlaufsbalken dargestellt. Werden die Aktivitäten losgelöst von dem tabellarischen Konstrukt des Gantt-Diagramms verwendet, so lässt sich das Diagramm auch in ein SB überführen (siehe Abb. 5). Die zeitliche Einteilung lässt sich in diesem Zusammenhang auf die horizontale Achse verlegen, welche die zeitliche Abfolge von einzelnen Aktivitäten darstellt.

Die Verwendung einer fixen zeitlichen Einteilung auf der horizontalen Achse ist hingegen für die Modellierung von Dienstleistungsprozessen schwierig, da somit keine Iterationen oder Rücksprünge modelliert werden können. Diese könnten somit nur innerhalb einer Aktivitätsverfeinerung oder gar nicht erfasst werden. 


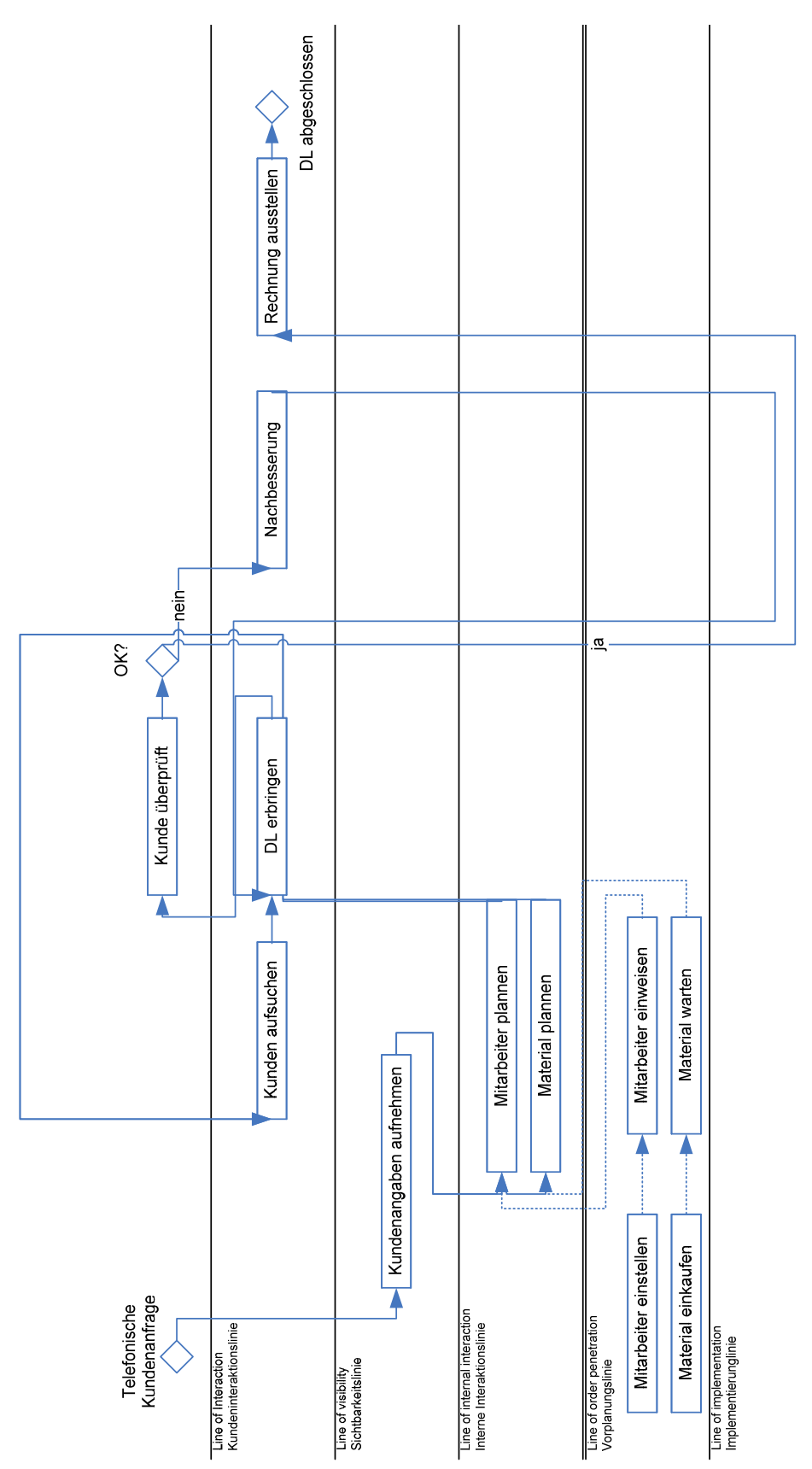

Abb. 5. Beispielprozess als Gantt-Diagramm in SB-Anordnung 


\section{Ereignisgesteruerte Prozessketten}

Die Modellierung von Prozessen, genauer von Geschäftsprozessen, mittels ereignisgesteuerter Prozessketten (EPK) ist weit verbreitet. Genauere Ausführungen zu EPK werden in (Scheer und Thomas 2005) gegeben. Nachfolgend wird der Beispielprozess in der EPK dargestellt. Bei einer EPK erfolgt die Modellierung in der Regel von oben nach unten beginnend mit einem Ereignis. Zwischen Ereignissen und Funktionen wechselnd wird der Beispielprozess mit dem Erbringen der Dienstleistung beendet. Zur Überführung des Prozesses in EPK-Notation in die SB-Anordnung muss eine einheitliche Modellierungsrichtung eingehalten werden. Unter Berücksichtigung des Service Blueprints ist in diesem Falle die horizontale Ausrichtung vorzuziehen. Somit besteht die Möglichkeit, nach Zuordnung der einzelnen Ereignisse und Funktionen zu den Ebenen des Service Blueprints, das überführte Modell aufzustellen (Abb. 6 verdeutlicht die Nutzung von EPK in einer SB-Anordnung).

Die EPK ermöglicht die Darstellung der Prozesse. Allerdings werden durch die Wechsel zwischen Funktionen und Ereignissen die Prozesse umfangreich und teilweise unübersichtlich. 


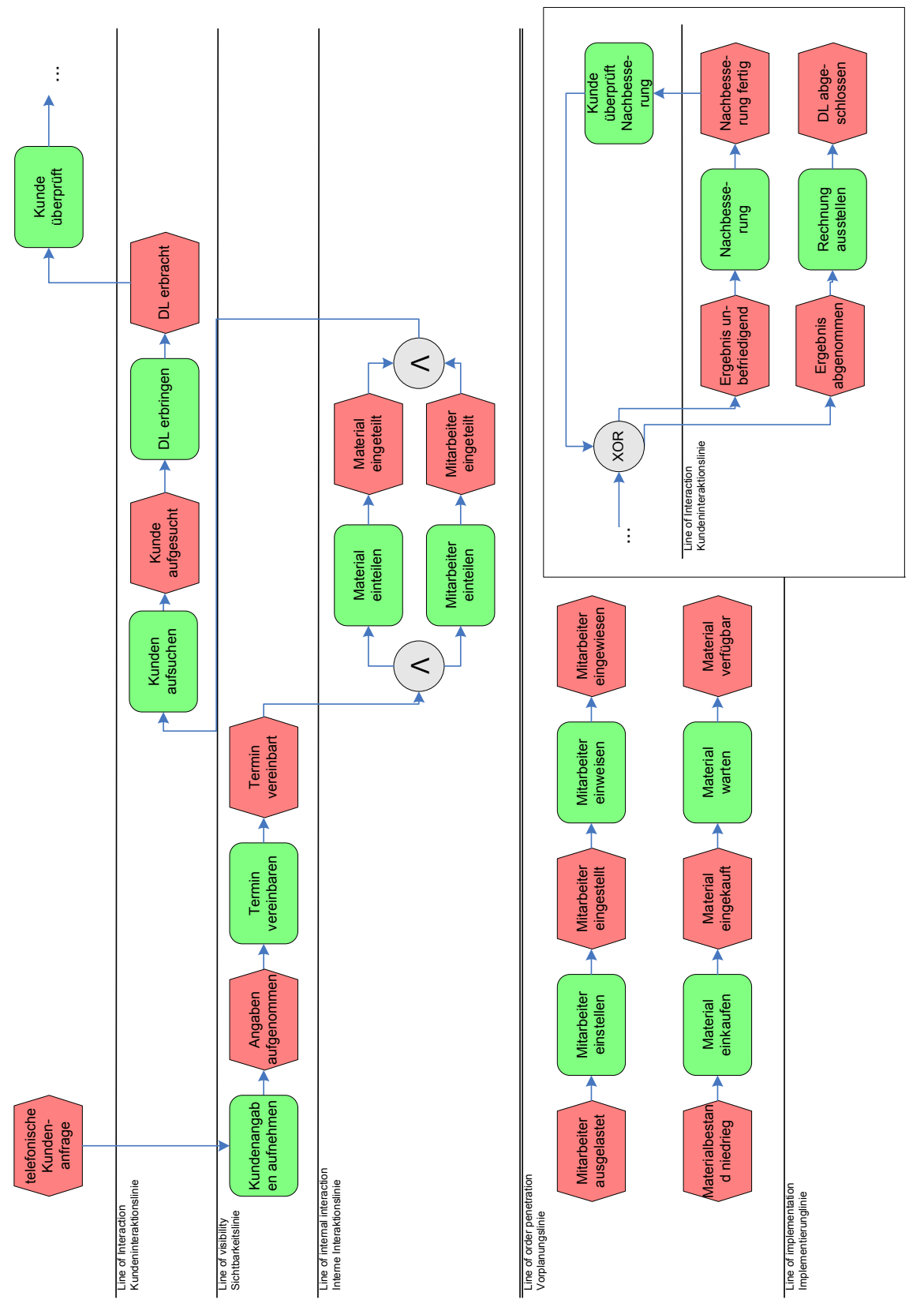

Abb. 6. Beispielprozess als EPK in SB-Anordnung 


\subsection{Bewertung}

Zusammenfassend lässt sich feststellen, dass mit Hilfe des SB sich die Kundeninteraktionen mit unterschiedlichen Notationen darstellen lassen. Die Interaktion mit dem Kunden als externer Faktor ist gerade bei einem Dienstleistungsprozess eine wichtige Eigenschaft. Weiterhin müssen die Immaterialität und das Uno-ActuPrinzip einer Dienstleistung im Dienstleistungsprozess berücksichtigt werden (Meffert und Bruhn 2000; Meyer 1992). Hinzu kommt, dass die Methoden die Berücksichtigung der technischen Entwicklung aufgreifen müssen. Die Entwicklungsstufen des Service Blueprints zeigen, dass die Technisierung auch in der Dienstleistungsentwicklung und somit der Dienstleistungsunterstützung Einzug gehalten hat.

Aus Sicht der Dienstleistungsprozesse und der Modellierung von Dienstleistungen ist zu prüfen, welche Aktivitäten innerhalb eines Modells dargestellt werden sollten. Das Service Blueprint erlaubt das Weglassen von einzelnen Ebenen. Somit wird eine kompakte Prozessdarstellung unterstützt. Dieses ermöglicht eine differenzierte Betrachtung von Leistungsprozess und Leistungspotenzial. Daher ist kritisch zu prüfen, welche Prozesse des Leistungspotenzials (z.B. Facility Aktivitäten ohne Bezug zum Leistungsprozess) mit in das Modell aufzunehmen sind.

Die Unterstützung für Dienstleistungsprozesse wird im SB durch Anlehnung an die Flussdiagramme am besten verdeutlicht. Die veranschaulichten verschiedenen Notationen, die mit dem SB zusammen Verwendung gefunden haben, zeigen den flexiblen Einsatz des Service Blueprints. Allerdings stellen die vorgestellten Notationen keine ausreichende Unterstützung für IT-gestützte wohnbegleitende Dienstleistungen dar. Folglich ist die Möglichkeit zu prüfen, welche aktuellen Notationen mit dem SB zu kombinieren sind, um der Technisierung von Dienstleistungen Rechnung zu tragen. Mit der Business Process Modeling Notation (BPMN) wird dies ermöglicht, da sowohl die fachliche als auch die technische Modellierung von Prozessen abgedeckt wird.

\section{Business Process Modeling Notation}

\subsection{Historie}

Die BPMN ist eine Modellierungssprache mit dem Fokus, sowohl fachliche als auch technische Modelle zu erstellen (OMG 2009). Die BPMN, die kurz nach dem Zusammenschluss der Business Process Modeling Initiative (BPMI) mit der Object Management Group (OMG) Mitte 2005 verabschiedet wurde, steht vor der Einführung der Version 2.0. Mit der BPMN scheint sich ein Standard für die Prozessmodellierung durchzusetzen, da bereits mehr als 60 Modellierungswerkzeuge die BPMN unterstützen (OMG 2009). Die BPMN 2.0 hat eine größere Umstellung erfahren und umfasst neben der grafischen Darstellung auch eine Serialisierung, die zur Überführung und Ausführung modellierter Prozesse in einer Systemumge- 
bung verwendet wird. Ist bei der Version 1.2 noch auf eine ausführbare Prozessbeschreibung wie z.B. BPEL (Business Process Execution Language) verwiesen worden, so wird in der Version 2.0 die Serialisierung mitspezifiziert. Somit ist eine Überführung von einem fachlichen zu einem technischen Modell möglich, welches anschließend von einem Business Process Management System (BPMS) ausgeführt werden kann.

Den Kern von BPMN bilden sequenzielle Abläufe von Prozessen, Ereignisse, Aktivitäten und Nachrichten. In Form von Diagrammen dargestellt kann der Benutzer sowohl auf hohem Abstraktionsniveau als auch auf einem sehr detaillierten Niveau Prozessmodelle formulieren (OMG 2004). Untersuchungen zeigen, dass die BPMN auf der Detailebene als Prozessnotation sowohl von Business Spezialisten als auch IT-Spezialisten vermehrt eingesetzt wird (camunda 2008) und detaillierte Prozesse als Grundlage zur systematischen Ausführung von Prozessmodellen verwendet werden (Siegel 2008).

\subsection{Methode}

Die BPMN verwendet eine einfache Syntax. Die Syntax umfasst, übergreifend über die Diagrammtypen, Basiselemente und erweiterte Elemente. Die erweiterten Elemente werden in diesem Zusammenhang nicht näher erläutert. Eine ausführliche Betrachtung alle Elemente wird in der Spezifikation (OMG 2007) gegeben. $\mathrm{Zu}$ den Basiselementen der BPMN, angelehnt an die Flussdiagramme, gehören (OMG 2007; White 2004):

- Fluss-Objekte:

- Ereignis (Kreis mit einfachen, doppelten oder dicken Kanten und Icon für den Event-Typ)

- Aktivität (Rechteck mit abgerundeten Ecken, Label und opt. Markern), auch geschachtelt für Subprozesse

○ Übergang/Gateway (Raute, wie sie auch in Ablaufdiagrammen verwendet wird), zur Spezifikation des Kontrollflusses

- Verbinder (Linienarten) für Sequenzfluss, Nachrichtenfluss und Assoziationen

- Pools und Lanes (rechteckige Zeichenbereiche) zur Darstellung von Ressourcen und Prozessbeteiligten

- Pools stellen einen eigenen Prozess von einer Ressourcen oder eines Prozessbeteiligten dar.

- Lanes sind Bestandteile eines Pools und somit abhängig von diesem. Sie stellen eine übersichtliche Unterteilung innerhalb eines Pools bereit.

- Weitere Artefakte (Daten, Gruppen, Text Anmerkungen) 
In der Abb. 7 wird die BPMN auf den Beispielprozess (vgl. Abb. 3) angewendet. Da es sich hierbei um eine einfache Darstellung handelt, sind keine Aktivitäten beispielsweise einer Organisationseinheit oder einem Standort zugeordnet und folglich werden keine Pools und Lanes verwendet. Zur Modellierung des Beispielprozesses werden Ereignisse (Start und Endereignis), Aktivitäten und Übergänge modelliert.

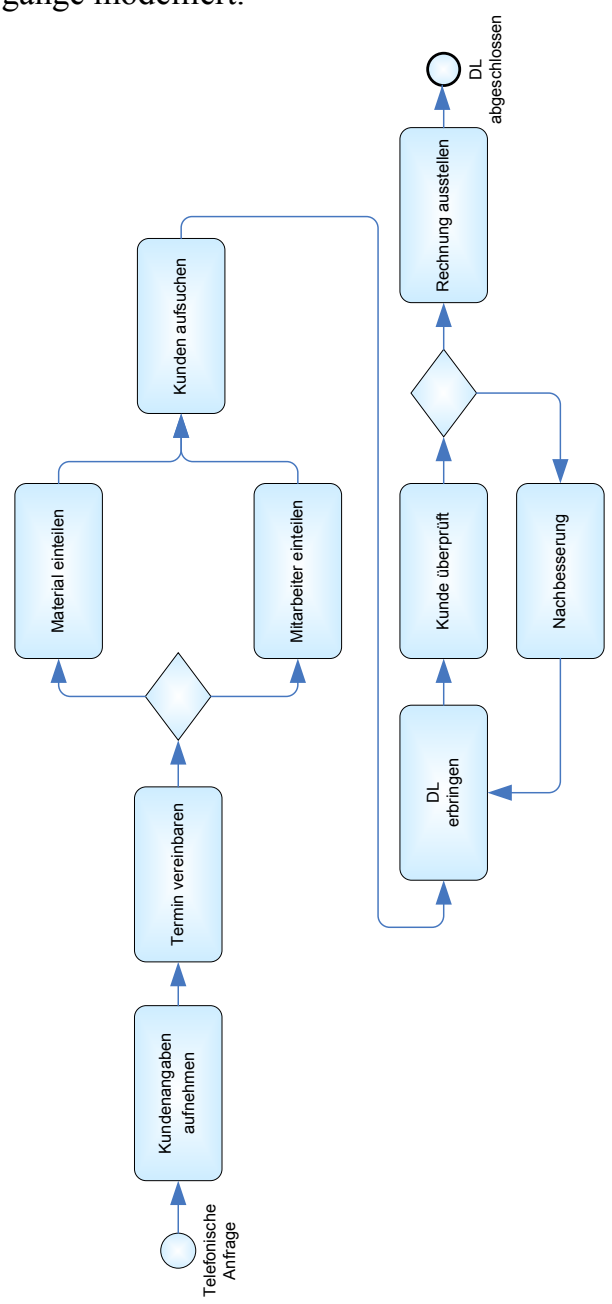

Abb. 7. BPMN Beispielprozess auf Basis des Anwendungsbeispiels 


\subsection{Bewertung}

Die BPMN ermöglicht mit ihren Aktivitäten, Ereignissen und Nachrichten eine Definition von systemgestützten als auch von manuell durchgeführten Aktivitäten. Modelle können mit der BPMN auf der fachlichen und auf der technischen Ebene erstellt werden. Einfache Modelle sind leicht und verständlich mit der BPMN zu modellieren und nachzuvollziehen. Auch bei komplexeren Modellen bietet die BPMN einen guten Überblick. Die hohe Anzahl von verfügbaren Werkzeugen zur Unterstützung der BPMN unterstreicht die Dominanz der Methode in der Anwendung. Des Weiteren ermöglicht die Ausführung von BPMN modellierten Prozessen in einem BPMS die Integration von IKT in den Dienstleistungsprozess, so dass die Technisierung in Dienstleistungen berücksichtigt wird. Dieses ist vor allem bei der Berücksichtigung von Sensoren und Aktoren innerhalb von IT-gestützten wohnbegleitenden Dienstleistungen wichtig.

Zur Modellierung von wohnbegleitenden Dienstleistungen bietet die BPMN mit ihren Pools und Lanes zwar eine hohe Zahl an Freiheitsgraden, und Pools und Lanes können sehr flexibel zur Abbildung von Organisationsstrukturen, Standorten oder Systemen eingesetzt werden. Dieses erfordert jedoch viel Kreativität und bietet keine ausreichende systematische Methode zur Modellierung von ITgestützten wohnbegleitenden Dienstleistungen. Im Folgenden wird daher eine Möglichkeit vorgestellt, wie mit Hilfe des BSBM, der Kombination von SB und BPMN, eine systematische Ausrichtung auf den Kunden ermöglicht wird.

\section{Methodischer Ansatz von BSBM}

\subsection{Einordnung}

BSBM kombiniert den Ansatz des SB mit der Notation der BPMN. Der Idee von Shostack folgend, kann die BPMN zur Modellierung von Service Blueprints eingesetzt werden, um die systematische Entwicklung von IT-gestützten wohnbegleitenden Dienstleistungen zu ermöglichen. Das SB unterstützt die kundenorientierte Modellierung von Dienstleistungen, welche von einem BPMS unterstützt ausgeführt werden können. Um die Ausführung auf einem BPMS zu erreichen wird die BPMN verwendet. Sie bietet mit den Pools und Lanes bereits eine mögliche Abbildung der Ebenen des Service Blueprints. Offen ist hingegen, welche Ebenen des Service Blueprints als Pool und welche als Lane dargestellt werden sollten. Grundsätzlich sind aufgrund der Spezifikationen der BPMN drei Varianten bei der Verwendung von BPMN mit SB möglich:

- Variante 1: Jeweils ein Pool für den Kunden und Dienstleister und die zugehörigen Ebenen werden als unterteilende Lanes dargestellt.

- Variante 2: Jede Ebene des Service Blueprints wird als eigenständiger Pool modelliert. 
- Variante 3: Die Ebenen des Service Blueprints werden als Lanes innerhalb eines Pools modelliert.

Diese Varianten besitzen unter Berücksichtigung der Modellierungsspezifikationen (z.B. Sequenz- und Nachrichtenfluss) der BPMN unterschiedliche Charakteristika. Bei der Verwendung des Service Blueprints muss in Abhängigkeit der Dienstleistung entschieden werden, welche Ebenen Verwendung finden. Nicht für alle Dienstleistungen werden alle Ebenen verwendet, da beispielsweise die Vorplanung für eine Dienstleistung nicht dargestellt werden soll bzw. notwendig ist.

\section{Beispieldienstleistung ,Vitalcheck“6}

Um die Technisierung in einem Dienstleistungsprozess zu konkretisieren, wird ein Beispiel verwendet, bei dem Sensoren als Informationslieferanten auf der Ebene der selbständig agierenden Kundenaktivitäten eingesetzt werden. Die betrachtete Dienstleistung ,Vitalcheck“ wird eingesetzt, um Bewohnern Sicherheit in der eigenen Wohnung zu geben. Im Falle eines Unfalls (z.B. Sturz) wird somit frühzeitig eine Kontaktperson benachrichtigt, dass keine Aktivität mehr stattfindet, obwohl der Bewohner zu Hause ist. Dieses wird dadurch gewährleistet, dass einzelne Aktivitäten (z.B. Bewegung, Öffnen von Türen, Fenstern oder Kühlschrank) ausgewertet werden. Kommt der Bewohner nach Hause, wird die Dienstleistung gestartet und regelmäßig in der Wohnung vorhandene Sensoren auf Ihren Status überprüft.

Finden regelmäßig Aktivitäten innerhalb der Wohnung statt ist alles in Ordnung. Bleiben hingegen Aktivitäten aus, obwohl der Bewohner anwesend ist, so informiert das System den Dienstleister. Dieser holt weitere Sensorinformationen aus der Wohnung ein, um die aktuelle Situation besser einschätzen zu können. Liegen alle Informationen vor, die eine Benachrichtigung notwendig machen, werden die Benachrichtigungen an vorab definierte Kontaktpersonen (z.B. Nachbarn, Angehörige) versendet. Mit der Benachrichtigung wird die Kontaktperson über die aktuelle Situation informiert und sucht ggf. den Bewohner auf. Kundensichtbare Aktivitäten (z.B. Installation, Einrichtung, Hilfestellungen) werden hier nicht betrachtet. Im Vorfeld sind mit dem Kunden der Leistungsumfang und die Einstellungsparameter zu definieren, damit eine problemlose Überführung, Durchführung und Dienstleistungserbringung realisiert werden kann.

\subsection{Variante 1 mittels Pools und Lanes}

Bei der Modellierung mittels SB wird die Interaktion des Kunden mit dem Dienstleister in den Vordergrund gestellt. Somit sind zwei Akteure am Dienstleistungsprozess beteiligt, die jeweils mit einem Pool voneinander getrennt dargestellt werden, der Kunde und der Dienstleister. Folglich werden bereits bei der Modellierung visuell die beiden Akteure voneinander getrennt. Die Ebenen des SB werden als Lanes in den Pools eingeordnet. Eine weitere Unterteilung (Lanes in Lanes) ist nicht vorgesehen, kann allerdings bei komplexeren Dienstleistungen in 
Form von „nested Lanes“ sinnvoll sein (Wolter und Schaad 2007). Die Dienstleistung „Vitalcheck“" wird in Abb. 8 in dieser Darstellungsvariante dargestellt.

In Abb. 8 werden die Nachrichtenaustausche zwischen dem Kunden und seiner Wohnung und dem Dienstleister verdeutlicht. Die Aufteilung der Aktivitäten, die vom Kunden und vom Dienstleister erbracht werden, wird durch die Einordnung zu einem Pool klar herausgestellt und es bleibt übersichtlich. Verschachtelte Lanes (z.B. bei der Übernahme von einzelnen Aktivitäten innerhalb einer Ebene) reduzieren die Übersichtlichkeit des Modells. Dieses ist bei komplexeren Dienstleistungen $\mathrm{zu}$ berücksichtigen.

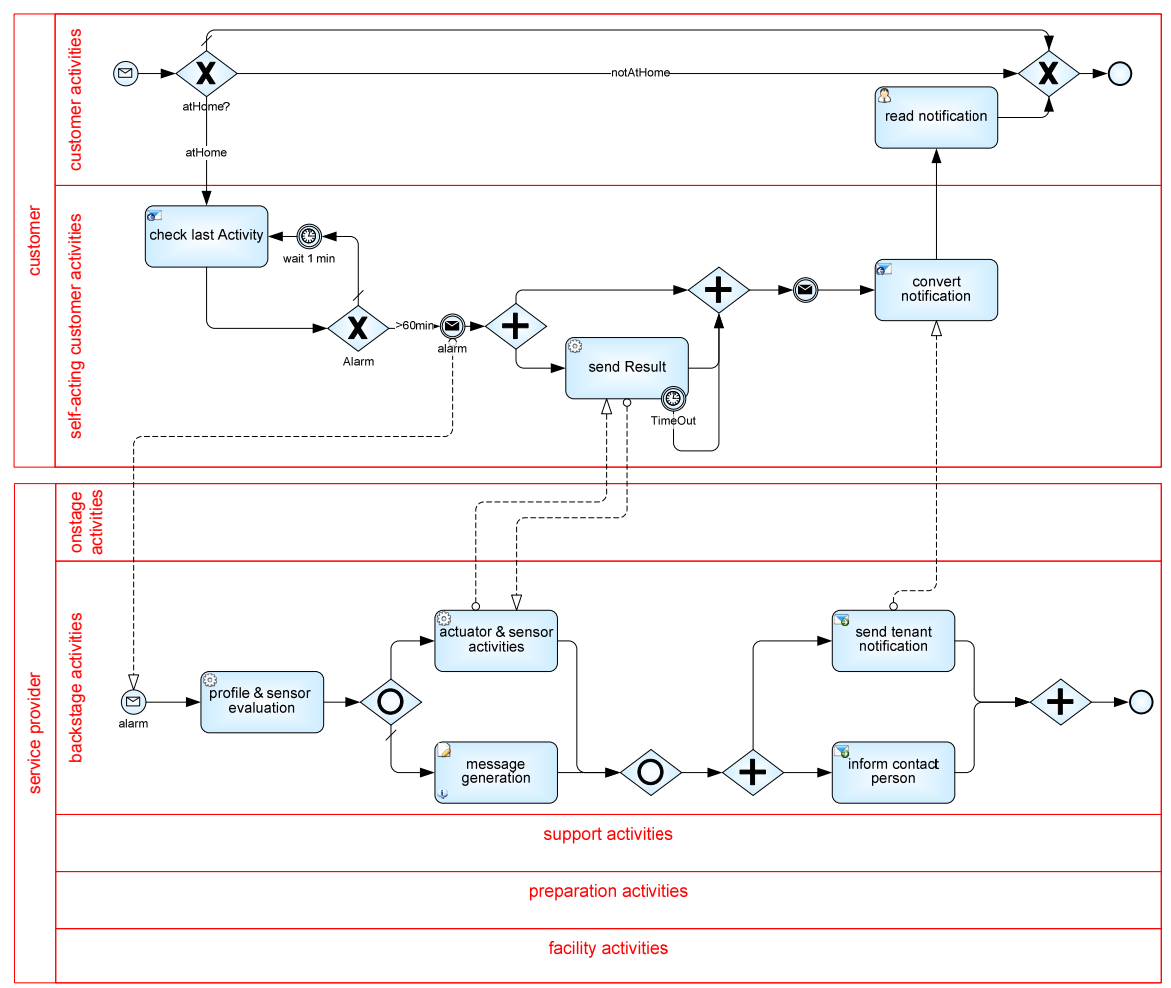

Abb. 8. Abbildung mit zwei Pools - für den Kunden und den Dienstleister

Werden die einzelnen Lanes zugeklappt dargestellt (z.B. facility activities, support activities), dann sind diese als Black Box zu betrachten. Diese Darstellung ist nur möglich, wenn keine Sequenzflüsse in diesen Lanes dargestellt werden. Folglich können diese Ebenen auch aus der Darstellung eliminiert werden. Um die Kommunikation nachzuvollziehen und gleichzeitig nicht in die eigenen Prozesse Einblick zu gewähren, wird zwischen öffentlichen und privaten Prozessen unterschieden. Dieses ist mit dem SB möglich, da die sichtbaren Aktivitäten (onstage 
activities) von den unsichtbaren Aktivitäten (backstage, support, preparation und facility activities) getrennt sind.

Durch Variante 1 werden Nachrichtenflüsse zwischen Dienstleister und Kunde sowie Sequenzflüsse beim Kunden und innerhalb des Dienstleisters unterstützt. Beim Kunden treten meist keine weiteren Nachrichtenflüsse auf. Sind weitere Nachrichtenflüsse hingegen beim Dienstleister notwendig, z.B. bei der Einbindung von Systemen, dann können diese nicht direkt in die angewendete Service Blueprint Struktur aufgenommen werden, sondern müssen als Sequenzflüsse innerhalb des Pools dargestellt werden.

\subsection{Variante 2 mittels Pools}

Bei der reinen Darstellung des Service Blueprints mittels Pools werden die einzelnen Ebenen des Service Blueprints jeweils durch einzelne Pools repräsentiert (siehe Abb. 9 mit dem Beispielprozess „Vitalcheck“). Die Übersicht zeigt bereits, dass bei der reinen Verwendung von Pools die Zuordnung der Ebenen zu Kunden- und Dienstleisteraktivitäten nicht mehr gegeben ist. Die einzelnen Pools sind eigenständig und agieren abhängig von den Nachrichtenflüssen miteinander.

Die Aufteilung zwischen öffentlichen und privaten Prozessen ist hier eindeutig an den Pools festzumachen, so dass hier die Pools eigenständig betrachtet werden können. Über die Integration von weiteren Lanes oder auch nested Lanes ist eine umfangreichere übersichtlichere Unterteilung möglich. Sequenzflüsse finden innerhalb der Pools statt, so dass in diesem Falle eine Kommunikation mittels Nachrichten umgesetzt werden muss.

Werden die Pools zugeklappt, so sind diese als Black Box zu sehen. Folglich werden nur die eingehenden und ausgehenden Nachrichtenflüsse zu dem Pool dargestellt, ohne einen direkten Bezug zu einer Aktivität zu verdeutlichen. Finden hingegen keine Nachrichtenflüsse mit den zugeklappten Pools statt, so können diese Pools auch entfallen (siehe Abb. 9 support, preparation und facility activities). 


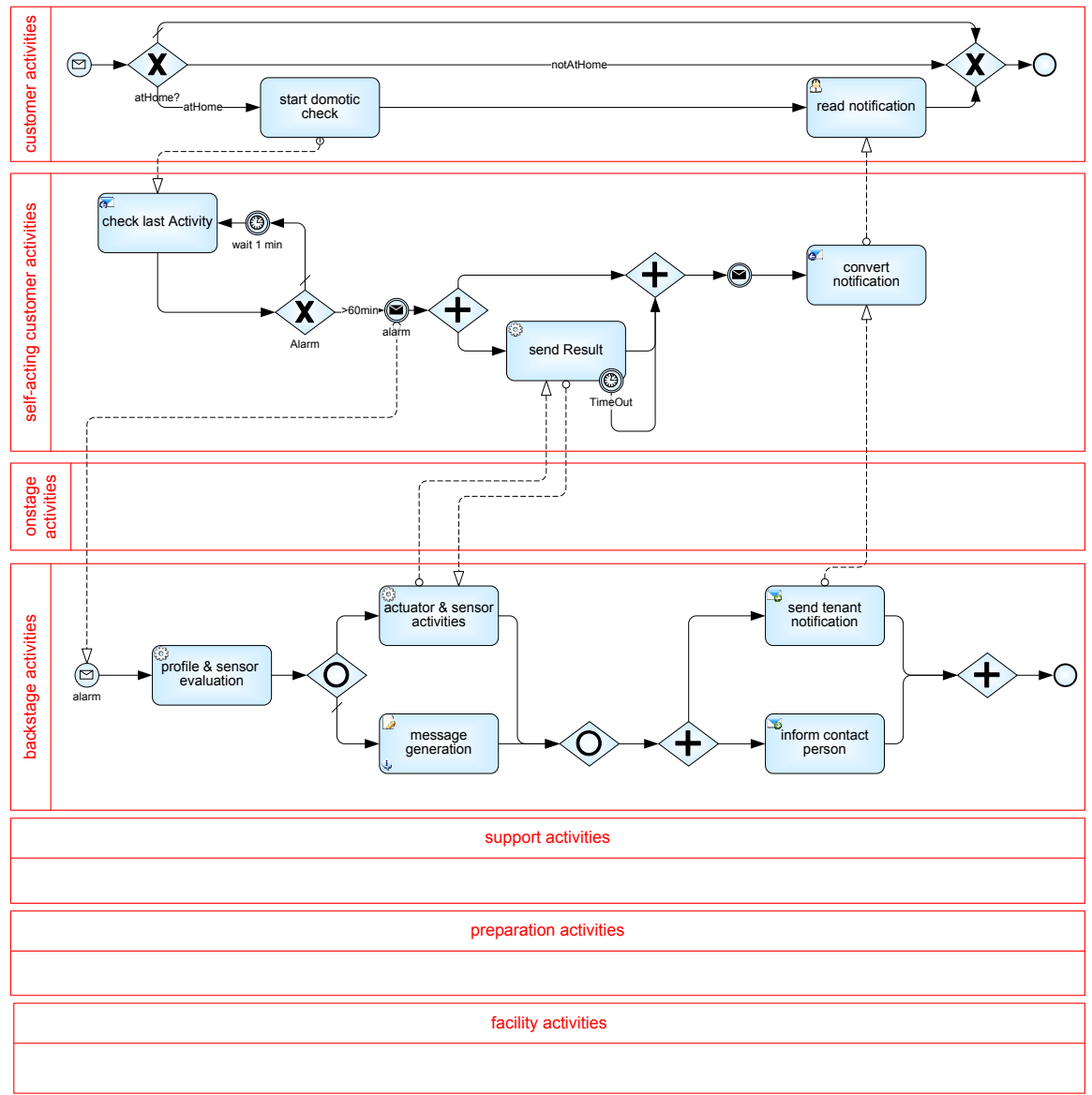

Abb. 9. Abbildung mit eigenen Pools - ein Pool je SB-Ebene

\subsection{Variante 3 mittels Lanes}

Bei der reinen Darstellung des Service Blueprints mittels Lanes werden die einzelnen Ebenen des Service Blueprints jeweils durch einzelne Lanes repräsentiert (siehe Abb. 10). Ein Pool ist bei dieser Darstellung nur optional einzuzeichnen. Ist ein Pool nicht integriert, dann ist das Modell implizit in einen Pool eingebettet, welcher nicht sichtbar ist. Zur besseren Darstellung ist das Einzeichnen des Pools von Vorteil. Aus der Abbildung wird deutlich, dass bei der Verwendung von Lanes eine offensichtliche Zuordnung der Ebenen zu Kunden- und Dienstleisteraktivitäten, vergleichbar mit der Variante 2, nicht mehr gegeben ist. Die einzelnen Lanes besitzen keine nachrichtenbasierte Kommunikation untereinander mehr, sondern sind über den Sequenzfluss miteinander verbunden. 


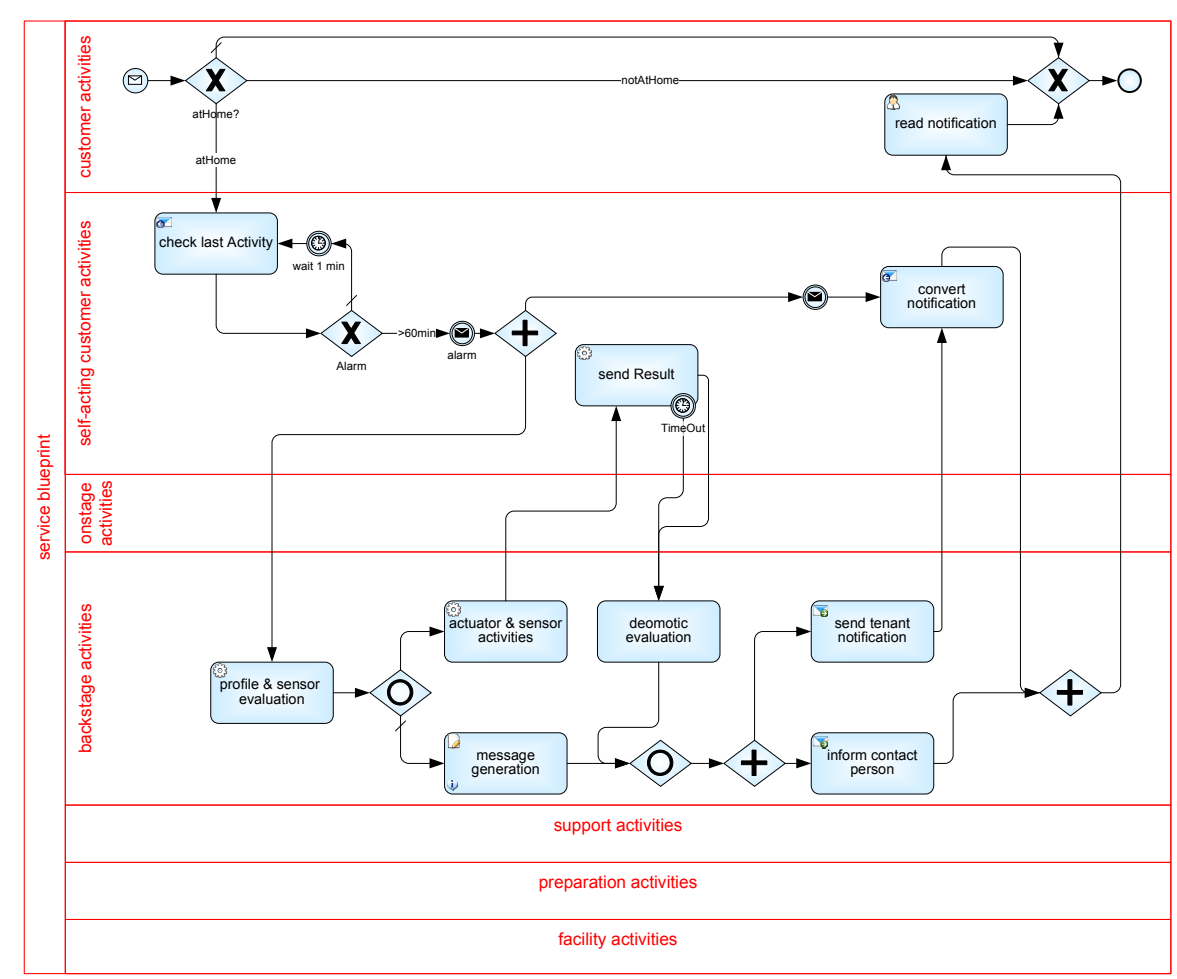

Abb. 10. Abbildung mit eigenen Lanes - eine Lane je SB-Ebene

Eine Aufteilung zwischen öffentlichen und privaten Prozessen ist zwar durch die Ebenenstruktur des Service Blueprints bei dieser Variante möglich, allerdings sind die Lanes nicht als Black Box darzustellen, weil sie einen Bestandteil des Sequenzflusses darstellen. Sind keine Aktivitäten innerhalb einer Ebene, sprich einer Lane vorgesehen, dann kann diese aus der Darstellung weggelassen werden.

Nested Lanes können innerhalb der Lanes verwendet werden. Die Integration von weiteren nested Lanes geht allerdings zu Lasten der Übersichtlichkeit.

\subsection{Kriterienkatalog zur Bewertung der Varianten}

Um die Varianten bewerten und vergleichen zu können, müssen Kriterien aufgestellt werden. Diese ermöglichen es, die Rahmenbedingungen für die Einsatzmöglichkeiten der beiden Varianten aufzuzeigen. Die Kriterien sind angelehnt an in der Literatur diskutierten Eigenschaften (Zeithaml et al. 2009; Fließ 2006). Des Weiteren sind Anforderungen in Projekten zum Service Wohnen, z.B. SmarterWohnenNRW (Draeger et al. 2008b) oder SmartLiving (Draeger et al. 2008a), zusammengefasst und aufgestellt worden. Bei den Forschungsprojekten wurden Dienstleistungen, unterstützt durch in der Wohnung verbaute Sensoren und Aktoren, den 
Bewohnern angeboten. Dabei wurden technische Dienste (reine Funktionalität innerhalb der Wohnung, z.B. automatische Herdabschaltung) und persönliche Dienstleistungen (kombinatorischer Einsatz von Sensoren bzw. Aktoren mit Dienstleistungen, z.B. Vitalcheck oder Menüservice) unterschieden. Im Folgenden werden wesentliche Kriterien, die zur Unterscheidung der Varianten herangezogen werden, aufgelistet und näher erläutert. Anschließend werden die beiden Varianten in einer Tabelle anhand der Kriterien eingestuft:

\section{- Kundeninteraktionspunkte}

Darstellung von Kundeninteraktionspunkten, teilweise als Integrativität bezeichnet (Engelhardt et al. 1993; Meffert und Bruhn 2000), an denen der Kunde die Qualität und die Außendarstellung des Dienstleisters wahrnimmt. Wichtig für die Modellierung ist, wie diese Kundeninteraktionspunkte einfach, verständlich und nachvollziehbar visualisiert werden können.

- Freiheitsgrade im Dienstleistungsprozess

Darstellung von einzelnen und gruppierten Organisationseinheiten (OE), Standorte oder Systemen, die Aufgaben innerhalb des Dienstleistungsprozesses übernehmen. Diese beeinflussen die Freiheitsgrade zur Modellierung der Dienstleistung.

- Berücksichtigung eigener Aufbauorganisation

Die Darstellung der internen Struktur, welche das Aufzeigen von involvierten OE, Standorten oder Systemen verdeutlicht. Diese können sowohl intern als auch extern sein.

- Berücksichtigung eigener Ablauforganisation

Die Darstellung der internen Abläufe, welche das Aufzeigen von involvierten OE, Standorten oder Systemen und deren Schnittstellen zur Nutzung innerhalb von Aktivitäten verdeutlicht. Diese können interne sowie externe Schnittstellen sein.

- Integration der Technisierung

Die Berücksichtigung der IKT als Bestandteil des Dienstleistungsprozesses ist für IT-gestützte Dienstleistungen wichtig. In der Anwendungsdomäne der wohnbegleitenden Dienstleistungen kommen hierfür Sensoren und Aktoren sowie weitere technische Komponenten in Betracht, die innerhalb einer Wohnung vorhanden sind. Die Integration von Systemen ist mit den „system tasks“ gegeben und unabhängig von verwendeten Pools und Lanes.

Diese Kriterien zeigen auf, dass unterschiedliche Aspekte zu berücksichtigen sind. Im Folgenden werden für die Kriterien die Einschätzungen der beiden Möglichkeiten gegeben und tabellarisch gegenübergestellt. 


\subsection{Bewertung}

In der ausgewählten Anwendungsdomäne der wohnbegleitenden Dienstleistungen lässt sich die Anwendung von BSBM in einer entsprechenden Umgebung verdeutlichen. Die Schnittstellen zwischen den einzelnen Pools bzw. Lanes zeigen aus den modellierten Dienstleistungen die Anforderungen an die Interaktionen auf.

Tabelle 1. Bewertung der Varianten anhand definierter Kriterien

\begin{tabular}{|c|c|c|c|}
\hline Kriterium & Variante 1 & Variante 2 & Variante 3 \\
\hline $\begin{array}{l}\text { Kundeninter- } \\
\text { aktionspunkte }\end{array}$ & $\begin{array}{l}\text { Die Kundeninterakti- } \\
\text { onspunkte werden } \\
\text { durch die Nachrichten- } \\
\text { flüsse zwischen den } \\
\text { beiden Pools darge- } \\
\text { stellt. }\end{array}$ & $\begin{array}{l}\text { Die Interaktionspunkte } \\
\text { sind zwischen den zwei } \\
\text { Pools „Kundenaktivitä- } \\
\text { ten“ und „Onstage Ak- } \\
\text { tivitäten“ mit Nach- } \\
\text { richtenflüssen darge- } \\
\text { stellt. }\end{array}$ & $\begin{array}{l}\text { Die Interaktionspunkte } \\
\text { stellen die Übergänge } \\
\text { des Sequenzflusses } \\
\text { zwischen den Lanes } \\
\text { „Kundenaktivitäten“ } \\
\text { und den „Onstage Ak- } \\
\text { tivitäten“ dar. }\end{array}$ \\
\hline $\begin{array}{l}\text { Freiheitsgrade } \\
\text { im Dienstleis- } \\
\text { tungsprozess }\end{array}$ & $\begin{array}{l}\text { OE, Standorte oder } \\
\text { Systeme können als } \\
\text { „nested Lane“ inner- } \\
\text { halb der Lanes des SB } \\
\text { dargestellt werden. Un- } \\
\text { tergruppen werden als } \\
\text { untergeordnete „nested } \\
\text { Lanes“ eingeordnet. }\end{array}$ & $\begin{array}{l}\text { OE, Standorte oder } \\
\text { Systeme werden als } \\
\text { Lane(s) innerhalb der } \\
\text { Pools dargestellt. Die } \\
\text { Untergruppen werden } \\
\text { als ,nested Lanes“ in } \\
\text { die Pools eingebettet. }\end{array}$ & $\begin{array}{l}\text { OE, Standorte oder } \\
\text { Systeme können als } \\
\text { „nested Lane“ inner- } \\
\text { halb der Lanes des SB } \\
\text { dargestellt werden. Un- } \\
\text { tergruppen werden als } \\
\text { untergeordnete „,nested } \\
\text { Lanes“ eingeordnet. }\end{array}$ \\
\hline $\begin{array}{l}\text { Berücksichti- } \\
\text { gung eigener } \\
\text { Aufbauorgani- } \\
\text { sation }\end{array}$ & $\begin{array}{l}\text { Für die interne Darstel- } \\
\text { lung werden nested } \\
\text { Lanes als Strukturmög- } \\
\text { lichkeit für den } \\
\text { Dienstleister ermög- } \\
\text { licht, wenn die SB- } \\
\text { Einordnung nicht aus- } \\
\text { reichen sollte. }\end{array}$ & $\begin{array}{l}\text { Bei den Pools können } \\
\text { übersichliche Lanes in- } \\
\text { tegriert und weitere } \\
\text { verschachtelte Lanes } \\
\text { zur Darstellung der } \\
\text { Aufbauorganisation } \\
\text { verwendet werden. }\end{array}$ & $\begin{array}{l}\text { Die Aufbauorganisati- } \\
\text { on ist in der reinen La- } \\
\text { nedarstellung mittels } \\
\text { nested Lanes hinzuzu- } \\
\text { fügen, wenn die SB- } \\
\text { Einordnung nicht aus- } \\
\text { reichen sollte. }\end{array}$ \\
\hline $\begin{array}{l}\text { Berücksichti- } \\
\text { gung eigener } \\
\text { Ablauforgani- } \\
\text { sation }\end{array}$ & $\begin{array}{l}\text { Für die interne Darstel- } \\
\text { lung werden Sequenz- } \\
\text { flüsse verwendet, so } \\
\text { dass hier direkt der } \\
\text { Kontrollfluss innerhalb } \\
\text { des Pools stattfindet. } \\
\text { Hingegen ist der Nach- } \\
\text { richtenaustausch, wel- } \\
\text { cher eine interne oder } \\
\text { externe Schnittstellen- } \\
\text { kommunikation unter- } \\
\text { stützt, nicht über die }\end{array}$ & $\begin{array}{l}\text { Die interne und externe } \\
\text { Kommunikation wird } \\
\text { über die Ebenen hin- } \\
\text { weg mittels Nachrich- } \\
\text { tenflüsse dargestellt. } \\
\text { Somit ist eine Darstel- } \\
\text { lung von Schnittstellen } \\
\text { anhand der Nachrich- } \\
\text { tenflüsse möglich. }\end{array}$ & $\begin{array}{l}\text { Da nur Sequenzflüsse } \\
\text { verwendet werden, ist } \\
\text { eine Trennung zwi- } \\
\text { schen interner und ex- } \\
\text { terner Kommunikation } \\
\text { schwer möglich. }\end{array}$ \\
\hline
\end{tabular}


Jochen Meis, Philipp Menschner und Jan Marco Leimeister

\begin{tabular}{|c|c|c|c|}
\hline Kriterium & Variante 1 & Variante 2 & Variante 3 \\
\hline & $\begin{array}{l}\text { Nachrichtenflüsse dar- } \\
\text { zustellen. }\end{array}$ & & \\
\hline $\begin{array}{l}\text { Integration der } \\
\text { Technisierung }\end{array}$ & $\begin{array}{l}\text { Die Schnittstellen für } \\
\text { die Sensoren- und Ak- } \\
\text { torenintegration sind } \\
\text { Bestandteil des Se- } \\
\text { quenzflusses und daher } \\
\text { nicht direkt erkennbar. }\end{array}$ & $\begin{array}{l}\text { Die Integration erfolgt } \\
\text { über die Verwendung } \\
\text { definierter Schnittstel- } \\
\text { len. Diese sind mittels } \\
\text { der Nachrichtenflüsse } \\
\text { auf der Ebene der selb- } \\
\text { ständig agierenden } \\
\text { Kundenaktivitäten } \\
\text { sichtbar. }\end{array}$ & $\begin{array}{l}\text { Die Schnittstellen zu } \\
\text { den selbständig agiren- } \\
\text { den Kundenaktivitäten } \\
\text { für die Sensoren- und } \\
\text { Aktorenintegration } \\
\text { sind Bestandteil des } \\
\text { Sequenzflusses und } \\
\text { daher nicht direkt er- } \\
\text { kennbar. }\end{array}$ \\
\hline
\end{tabular}

Aus der Gegenüberstellung der drei Varianten und der Bewertung der einzelnen Kriterien lässt sich feststellen, dass keine der Varianten für alle Arten von Dienstleistungen am besten geeignet ist. Lediglich die Variante 3 bietet keine Vorteile gegenüber dem Einsatz von Variante 1 bzw. Variante 2. Da nur Lanes verwendet werden, ist die Aufteilung zwischen Kunde und Dienstleister nicht sofort ersichtlich. Auch werden keine Schnittstellen für die externen und internen Interaktionen explizit berücksichtigt. Daher sind Variante 1 und Variante 2 der dritten Variante zu bevorzugen. Darüber hinaus hängt es vom speziellen Charakter der jeweiligen Dienstleistung ab, welche der beiden Variante zu bevorzugen ist. Handelt es sich dabei um einen zentralisierten Anbieter, bei dem alle Aktivitäten zentral organisiert sind, empfiehlt sich die Variante 1, da die Dienstleisteraktivitäten eng miteinander verbunden sind. Weist hingegen die interne Struktur des Dienstleisters eine sehr stark dezentral und serviceorientierte Struktur auf, dann empfiehlt sich die Darstellung mittels eigener Pools, da die Nachrichtenflüsse die Serviceschnittstellen zwischen den Aktivitäten gut aufzeigen. Ist hingegen eine Dienstleistung an den Kundeninteraktionspunkten sehr personalintensiv und verwendet dabei nur wenig IKT, sollte Variante 1 eingesetzt werden, da somit die Fokussierung auf die Kundeninteraktion gelegt wird. Die Verifikation des Einsatzes beider Varianten für IT-gestützte wohnbegleitende Dienstleistungen abhängig von speziellen Charakteristika muss anhand von Kriterien geprüft werden. Diese Kriterien sind noch zu ermitteln, so dass eine Bewertung und Überprüfbarkeit der ausgeführten Hypothesen vorgenommen werden kann.

Die Vorteile durch BSBM resultieren aus den Stärken der verwendeten Methoden. Durch das SB wird die Sichtbarkeit gegenüber dem Kunden explizit dargestellt und kann mittels der BPMN auch visualisiert werden. Öffentliche Aktivitäten werden durch die SB Ebenen von privaten Aktivitäten getrennt. Zusätzlich werden somit die Leistungen, an denen die Kundenmitwirkung notwendig ist, herausgestellt. Folglich sind an diesen Punkten die Kundeninteraktionen definiert, die die Ergebnisse und die Erwartungen an die Dienstleistung herausstellen. Des Weiteren stehen für die BPMN mehr als 60 Werkzeuge zur Verfügung. Die Modelle 
können auf Grund der Standardisierung der BPMN zu einem BPMS überführt und dort systemgestützt ausgeführt werden.

Zu den Nachteilen gehören, dass die Übersichtlichkeit bei komplexeren Dienstleistungsprozessen mit verschachtelten OE, Standorten oder Systemen reduziert wird. Dieses kann durch eine gezielte Aufteilung in Haupt- und Subprozesse vermieden werden. Abschließend ist festzustellen, dass die BPMN 2.0 noch nicht von allen Werkzeugen komplett unterstützt wird und somit auch eine Integration in BPMS noch nicht durchgehend gewährleistet ist. Folglich ist auch der werkzeugübergreifende Modellaustausch noch nicht vollständig möglich.

\section{Zusammenfassung und Ausblick}

Die aufgezeigten Potenziale bei der Kombination von SB und BPMN sind erkennbar. Anhand des Beispiels konnten Variante 1 und 2 zur Modellierung verwendet werden. Der Varianteneinsatz von Pools und Lanes gegenüber der reinen Pool-Verwendung ist im Einzelfall von der betrachteten Dienstleistung, ihrer Komplexität und der verwendeten Systemlandschaft abhängig. Die reine Verwendung von Lanes ist hingegen für BSBM nicht vorteilhaft.

Anhand eines Proof-of-Concept werden im weiteren Forschungsverlauf verschiedene IT-gestützte wohnbegleitende Dienstleistungen betrachtet und mit der Variante 1 und Variante 2 modelliert. Anschließend lässt sich die Überprüfung der Hypothesen und die Einstufung, wann welche der beiden Möglichkeiten bevorzugt einzusetzen ist, besser herausstellen. Verschiedene Werkzeuge unterstützen bereits die Modellierung von Prozessen, in der Regel Geschäftsprozesse, die allerdings nicht immer den Dienstleistungscharakter fokussieren. Somit lässt sich mit der Kombination von SB und BPMN eine Werkzeugunterstützung aufzeigen, welche eine spätere IT-Unterstützung für Dienstleistungen vorbereitet. Ein wichtiger Aspekt ist hierbei die Berücksichtigung von umfangreichen Sensoren und Aktoren oder weiteren technischen Komponenten (z.B. telemedizinische Geräte) innerhalb der Dienstleistung. Diese sollten unabhängig von einem installierten Gebäudeautomationssystem und Infrastruktur mitmodelliert werden können.

Als nächstes gilt es, einen Wirksamkeitsnachweis der vorgestellten Methode anhand von weiteren IT-gestützten wohnbegleitenden Dienstleistungen aus der Praxis zu liefern. Hierzu sind am Markt verfügbare IT-gestützte Dienstleistungen zu betrachten und mit der BSBM zu modellieren und anschließend zu bewerten.

\section{Literaturverzeichnis}

Berekoven L (1974) Dienstleistungsbetrieb - Wesen - Struktur Bedeutung. Wiesbaden Beuerle I, Petter S (2008) Sozialmanagement in Wohnungsunternehmen. 2. Aufl., Hammonia $\mathrm{GmbH}$, Hamburg

Böhmann T (2004) Modularisierung von IT-Dienstleistungen. Wiesbaden 
Bullinger HJ, Scheer AW (2006) Service Engineering - Entwicklung und Gestaltung innovativer Dienstleistungen. In: Bullinger HJ, Scheer AW (Hrsg): Service Engineering. Springer, Berlin

Camunda (2008) BPM-Pakete haben Nachholbedarf. openPR.de. Berlin, camunda service GmbH. 20-4-2008

Draeger J, Meis J, Schöpe L (2008) Smart Living HealthCare in den eigenen vier Wänden. In: Ambient Assisted Living - 1. Deutscher Kongress mit Ausstellung 2008, E.I.V. Verband der Elektrotechnik. VDE, Berlin, S. 337-342

Draeger J, Meis J, Schöpe L, Wojciechowski M (2008) SmarterWohnenNRW - Realisierung IT-gestützter Mehrwertdienste für den wohnungswirtschaftlichen Massenmarkt. In: Software Engineering 2008 - Fachtagung des GI-Fachbereichs Softwaretechnik. Bonn, S. 301

Engelhardt WH, Kleinaltenkamp M, Reckenfelderbäumer M (1993) Leistungsbündel als Absatzobjekt: Ein Ansatz zur Überwindung der Dichotomie von Sach- und Dienstleistungen. In: Zeitschrift für die betriebswirtschaftliche Forschung 45, 5, S. 395-426

Fließ S (2006) Prozessorganisation in Dienstleistungsunternehmen. Stuttgart

Fließ S, Kleinaltenkamp M (2004) Blueprinting the service company: Managing service processes efficiently. In: Journal of Business Research, 57, 4, S. 392-404

Fließ S, Lasshof B, Mekel M (2004) Möglichkeiten der Integration eines Zeitmanagements in das Blueprinting von Dienstleistungsprozessen. Douglas-Stiftungslehrstuhl für Dienstleistungsmanagement

Hilke W (1989) Grundprobleme und Entwicklungstendenzen des DienstleistungsMarketing. In: Hilke W (Hrsg): Dienstleistungs-Marketing. Wiesbaden, S. 5-44

Hohm D, Jonuschat H, Scharp M, Scheer D, Scholl G (2004) Innovative Dienstleistungen „rund um das Wohnen“ professionell entwickeln - Service Engineering in der Wohnungswirtschaft. GdW Bundesverband deutscher Wohnungsunternehmen e.V., 2004

Hsieh YH, Yuan ST (2010) A S-D Logic Based Approach to Input-Output Analysis for Technology Spillover. In: HICSS2010, Computer Society Press

Kingman-Brundage J (1989) The ABC's of Service System Blueprinting - Designing a Winning Service Strategy. Chicago

Kleinaltenkamp M (2008) Blueprinting - Grundlage des Managements von Dienstleistungen. In: Woratschek H (Hrsg): Neue Aspekte des Dienstleistungsmarketing - Konzepte für Forschung und Praxis. Wiesbaden, S. 3-28

Leimeister JM, Glauner C (2008) Hybride Produkte - Einordnung und Herausforderungen für die Wirtschaftsinformatik. In: Wirtschafsinformatik, 50, 3

Maleri R. (1997) Grundlagen der Dienstleistungsproduktion. Berlin

Meffert H, Bruhn M (2000) Dienstleistungsmarketing - Grundlagen, Konzepte, Methoden. Gabler, Wiesbaden

Meffert H, Bruhn M (2008) Dienstleistungsmarketing - Grundlagen, Konzepte, Methoden. 5. Aufl., Gabler, Wiesbaden

Meiren T, Hofmann HR, Klein L (1998) Vorgehensmodelle für das Service Engineering. In: Information Management \& Consulting, Sonderausgabe Service Engineering, 13, S. $20-25$

Meis J, Draeger J (2007) Modelling automated service orchestration for IT-based homeservices. In: International Conference on Service Operations and Logistics, and Informatics 2007. IEEE, Philadelphia, USA, S. 155-160 
Meis J, Schöpe L (2006) ServiceDesign with the Service Blueprint. In: International Conference on Software Engineering \& Knowledge Engineering. Knowledge Systems Institute Graduate School, San Francisco, USA, S. 708-713

Meyer A (1992) Dienstleistungs-Marketing: Erkenntnisse und praktische Ergebnisse. München, Augsburg

OMG (2008) Business Process Modeling Notation (BPMN) Information. http://www.bpmn.org/Documents/FAQ.htm Abrufdatum: 23. April 2008

OMG (2007) Business Process Model and Notation (BPMN) 2.0 - RFP. Request for Proposal. 5-6-2007

OMG (2009) BPMN Information. http://www.bpmn.org/ 26-12-2009

Philpott J (2000) Weightlessness and the political economy of service sector job creation. In: European Commission (ed): The job creation potential of the service sector in Europe. Luxembourg, S. 268-286

Pötzsch O, Sommer B (2006) Bevölkerung Deutschlands bis 2050. Statistisches Bundesamt - Pressestelle Wiesbaden, Wiesbaden

Rai A, Sambamurthy V (2006) The Growth of Interest in Services Management - Opportunities for Information Systems Scholars. In: Information Systems Research, 17, 4, 2006

Riedl C, Leimeister JM, Krcmar H (2009) New Service Development for Electronic Services - A Literature Review. In: Americas Conference on Information Systems (AMCIS‘09), San Francisco

RWI (2008) Potenziale des Dienstleistungssektors für Wachstum von Bruttowertschöpfung und Beschäftigung. Essen

Scharp M, Halme M, Jonuschat H (2004) Nachhaltige Dienstleistungen der Wohnungswirtschaft. 9. Aufl., IZT - Institut für Zukunftsstudien und Technologiebewertung, Berlin

Scheer AW, Thomas O (2005) Geschäftsprozessmodellierung mit der ereignisgesteuerten Prozesskette. In: Das Wirtschaftsstudium, 8, 9, S. 1069-1079

Schneider K, Thomas O (2003) Kundenorientierte Dienstleistungsmodellierung mit Ereignisgesteuerten Prozessketten. In: Nüttgens M, Rump FJ (Hrsg): GI-Arbeitskreis Geschäftsprozessmanagement mit Ereignisgesteuerten Prozessketten, S. 87-93

Schneider K, Wagner D, Behrens H (2003) Vorgehensmodelle zum Service Engineering. In: Bullinger HJ, Scheer AW (Hrsg): Service Engineering - Entwicklung und Gestaltung innovativer Dienstleistungen. Springer, Berlin, S. 117-141

Shostack GL (1984) Designing Services that deliver. In: Harvard Business Review, 62, 1, S. $133-139$

Siegel J (2008) BPMN unter der Lupe. In: omg-kolumne

White SA (2004) Introduction to BPMN. IBM Cooperation

Wolter C, Schaad A (2007) Modeling of Task-Based Authorization Constraints in BPMN. In: Alonso P, Dadam P, Rosemann M (eds): BPM 2007. Springer, Berlin, S. 64-79

Zeithaml VA, Bitner MJ, Gremler DD (2009) Services Marketing - Integrating the Customer Focus Across the Firm, 5th ed., McGraw-Hill Education, Singapore

Zink KJ, Eberhard DB (2009) Typologisierung von Dienstleistungen. In: Zink KJ (ed): Personal- und Organisationsentwicklung bei der Internationalisierung von industriellen Dienstleistungen. Physica, Heidelberg 